Ana Patricia Pabón Mantilla

Universidad Autónoma de Bucaramanga

Óscar Andrés Upegui Toledo 2

Universidad Autónoma de Bucaramanga

Jessica Johanna Archila Julio ${ }^{3}$

Universidad Autónoma de Bucaramanga

María Angélica Otero González Universidad Autónoma de Bucaramanga

\section{El acceso a las técnicas de reproducción asistida como una garantía de los derechos sexuales y reproductivos: la jurisprudencia de la Corte Constitucional a la luz del Derecho Internacional de los Derechos Humanos*}

The access to the technologies of reproduction represented as a guarantee of the sexual and reproductive rights: the jurisprudence of the Constitutional Court in the light of the International Law of the Human Rights

Recibido: 18 de abril de 2016 / Aceptado: 27 de julio de 2016

http://dx.doi.org/ 10.17081/just.22.31.2605

Palabras clave:

Derechos Humanos,

Derechos Sexuales y Reproductivos, Enfoque de los derechos, Infertilidad, Jurisprudencia constitucional,

Técnicas de reproducción asistida.

Key words:

Human rights,

Sexual and reproductive Laws, Approach of the rights, Unfertility,

Constitutional jurisprudence, Technologies of assisted reproduction.

\section{Resumen}

El presente artículo pretende mostrar al lector cómo la jurisprudencia de la Corte Constitucional colombiana sobre el acceso a Técnicas de Reproducción Asistida, de manera tardía e influenciada por el desarrollo de los derechos sexuales y reproductivos en el ámbito del Derecho Internacional de los Derechos Humanos, se enmarca en un precedente de gran valor para su protección en el ámbito interno al replantear la discusión sobre la afectación a la autonomía y salud reproductiva por la inaccesibilidad a dichos servicios desde el enfoque de los derechos, apartándose de la concepción casi inmutable que mantuvo por 15 años sobre la efectividad de los derechos fundamentales basada en una clasificación histórico-clásica.

\section{Abstract}

This article seeks to show the reader how the Colombian Constitutional Court case-law about access to Assisted Reproduction Technology late and influenced by the sexual and reproductive rights development in the International Human Rights Law, sets a precedent of great value for its protection in the national judicial decisions since the discussion about the reproductive health and autonomy infringement because of inaccessibility to those services has been reconsidered from a rights-based perspective, giving up an almost immutable conception that was supported for 15 years about fundamental rights effectivity based on a classic-historical classification.

Referencia de este artículo (APA): Pabón, A., Upegui, O., Archila, J. \& Otero, M. (2017). El acceso a las técnicas de reproducción asistida como una garantía de los derechos sexuales y reproductivos: la jurisprudencia de la Corte Constitucional a la luz del Derecho Internacional de los Derechos Humanos. En Justicia, 31, 171-187. http://dx.doi. org/ 10.17081/just.22.31.2605

\footnotetext{
* Artículo de reflexión resultado del trabajo de investigación "Acceso a técnicas de reproducción asistida mediante el sistema de salud colombiano", vinculado al Grupo de Investigación en Teoría del Derecho y Formación Jurídica, Categoría C de Colciencias, avalado por la Universidad Autónoma de Bucaramanga, UNAB.

1 Doctora en Derecho. Magíster en Hermenéutica Jurídica y Derecho. Profesora Asociada de la Facultad de Derecho de la Universidad Autónoma de Bucaramanga, UNAB, y de la Escuela de Fílosofía de la Universidad Industrial de Santander, UIS, Colombia Vinculada al Grupo de Investigación Teoría del Derecho y Formación Jurídica. apabon742@unab.edu.co. https://orcid.org/0000-0002-2550$135 \mathrm{X}$

Abogado Universidad Autónoma de Bucaramanga, UNAB, Colombia. oupegui33@unab.edu.co

3 Abogada Universidad Autónoma de Bucaramanga, UNAB, Colombia. jarchila5@unab.edu.co

4 Abogada Universidad Autónoma de Bucaramanga, UNAB, Colombia. motero20@unab.edu.co
} 


\section{INTRODUCCIÓN}

En distintas regiones del mundo se estima que hay cerca de $15 \%$ de parejas con problemas de fertilidad (Luna, 2013, p.35). Este hecho ha motivado que los problemas de fertilidad se valoren desde la perspectiva de la salud pública, dadas las implicaciones que tiene dicha circunstancia para el concepto de salud integral (McDonald, 2004). Las Técnicas de Reproducción Asistida (TRA), como recurso para superar la imposibilidad de estas parejas en edad fértil de concebir, implican altas erogaciones de dinero que no siempre pueden ser pagadas por las familias que requieren dichos tratamientos.

En Colombia el legislador no ha regulado la forma en que el sistema de salud debe atender las solicitudes para acceder a las distintas técnicas de reproducción asistida. El Sistema General de Seguridad Social en Salud ha excluido del Plan Obligatorio de Salud estos procedimientos destinados a garantizar la salud sexual y reproductiva de los y las afectadas.

En la actualidad, el derecho a la salud ha sido reconocido por la Corte Constitucional como un derecho autónomo, como parte de la necesidad de proteger la dignidad humana (Cárdenas, 2013). Este reconocimiento ha sido el producto de una ardua labor interpretativa de la Corte Constitucional, que en sus inicios mantenía una clásica división de derechos fundamentales según su ubicación y progresivamente, al incorporar el criterio de conexidad fue extendiendo la protección del derecho a la salud hasta la actual tesis que permite defender la salud como derecho constitucional fundamental autónomo.
Las familias afectadas por problemas de fertilidad recurrieron en algunas oportunidades a la acción de tutela como mecanismo para garantizar la protección de su derecho a la salud integral. El análisis de las decisiones de la Corte permite identificar tres etapas en la línea de decisiones de estos casos, en la primera la Corte niega el amparo por considerar que no es un derecho susceptible de protección por vía de tutela al no generarse una violación a la salud e integridad. En una segunda etapa la Corte protegió el derecho de las personas a quienes las EPS ya habían iniciado un tratamiento y habían generado una expectativa con ello. $\mathrm{Y}$ en una tercera etapa, como sentencia hito se encuentra la Sentencia T-274 de 2015, en donde la Corte protege los derechos de los solicitantes y fija reglas para garantizar su derecho.

El artículo presente, busca reflexionar acerca de la coherencia de la decisión de la Corte de 2015 con el marco de protección del Derecho Internacional de los Derechos Humanos frente a la garantía de los derechos sexuales y reproductivos, el problema del cual se partió consistió en determinar si ¿es procedente la acción de tutela como mecanismo para garantizar el acceso a las técnicas de reproducción asistida? Se defenderá la tesis de que la Corte estaba en mora de armonizar las subreglas constitucionales en materia de protección al derecho a la salud en el caso del acceso a técnicas de reproducción asistida, y que con la Sentencia de 2015 no solo unifica su jurisprudencia con la protección de la salud de forma integral, sino que además es coherente con las orientaciones que en esta materia en 
relación con los derechos reproductivos se fijan como estándares en el orden mundial.

Para abordar el problema propuesto y defender la hipótesis de trabajo, metodológicamente se parte de un análisis documental de tipo cualitativo con el fin de describir los argumentos que deben tenerse en cuenta en el abordaje del problema. Para sustentar la hipótesis de trabajo se analizarán a nivel doctrinario, los argumentos que permiten justificar, desde el enfoque de los derechos, la premisa de que la desprotección de los derechos sexuales y reproductivos acarrea serias violaciones al derecho a la libre opción de la maternidad y la paternidad; posteriormente se analizará la normatividad en el campo del Derecho Internacional de los Derechos Humanos y finalmente a nivel jurisprudencial, partiendo de la técnica de análisis dinámico de jurisprudencia, se valorarán las respuestas de la Corte Constitucional frente al problema de la procedencia de la acción de tutela para acceder a técnicas de reproducción asistida, concentrando el análisis en la decisión del 2015. El análisis de los documentos se hará desde una perspectiva crítica, lo que permitirá presentar a modo de conclusión, una valoración de la reciente decisión de la Corte desde la mirada del Derecho Internacional de los Derechos Humanos.

\section{La protección de los derechos sexuales y} reproductivos como garantía fundamental para el ejercicio de los derechos

El derecho a la salud, de manera amplia, debe entenderse como la garantía del bienestar corporal, mental y social. Dentro de este con- cepto se encuentra la salud reproductiva, que a su vez está ligada al disfrute de otros derechos. Así lo han entendido diversos sectores de la doctrina, para quienes

Los derechos reproductivos son derechos humanos que esencialmente se fundamentan en los principios de igualdad, autodeterminación y dignidad humana. Comprenden el derecho de los individuos a decidir de forma libre el número y espaciamiento entre los hijos, así como a tener la información y medios para ejercer esta autonomía (Arango, 2013 p.211).

La Constitución Política de Colombia en su artículo 42 consagra el derecho a la autonomía reproductiva al señalar que "la pareja tiene derecho a decidir libre y responsablemente el número de sus hijos". En este mismo artículo la Constitución le asegura una especial protección a la familia, reconociendo con ello su papel fundante en la sociedad.

En virtud de la protección especial a la maternidad y a la paternidad se ha desarrollado una valiosa jurisprudencia que permite afirmar la protección especial que debe asegurar el Estado colombiano a las decisiones que los asociados tomen frente a este tema, como parte del libre desarrollo de la personalidad, derecho que "se manifiesta singularmente en la definición consciente y responsable que cada persona puede hacer frente a sus propias opciones de vida y a su plan como ser humano, y colectivamente, en la pretensión de respeto de esas decisiones por parte de los demás miembros de la sociedad" (C. Const Sentencia T-668 de 2005).

La opción libre por la maternidad y la pa- 
ternidad está protegida por el derecho a la autodeterminación reproductiva, a la libertad y la garantía a la igualdad como derecho fundamental. Esta consagración constitucional faculta a los asociados a exigir del Estado la garantía a la igualdad material, lo cual supone ejecutar acciones para su efectiva realización. Esto implica que en situaciones de desventaja, el Estado deba realizar acciones para que las personas en estado de vulnerabilidad puedan restablecer el ejercicio de sus derechos, y en el caso de la maternidad y paternidad, para que no existan desestímulos ilegítimos a optar por el desarrollo de estas opciones de vida.

La imposibilidad de ser madre o padre limita las libertades y el derecho al libre desarrollo de la personalidad, así como el efectivo goce de los derechos sexuales y reproductivos, circunstancias que hacen necesaria la intervención del Estado como garante de la protección efectiva de los derechos de quienes no pueden disponer de los medios para asegurar su realización. Los derechos sexuales y reproductivos "parten de la libertad (en especial, de la libertad sexual) y requieren la igualdad (el piso común de la igualdad de información y acceso a los servicios de salud)" (Lamas, 2001, p.237).

La salud sexual y reproductiva permite la realización del proyecto de vida de las personas que desean ser padres. Quienes tienen problemas de salud que les impiden optar libremente por la maternidad o paternidad ven truncado dicho proyecto y con ello su realización personal. Esta situación afecta en mayor medida a quienes no pueden acceder a las técnicas de reproducción asistida como mecanismo para tratar su afección de salud por razones económicas. En esa medida se justifica reflexionar acerca de las obligaciones del Estado frente a la garantía de los derechos de las personas afectadas en su salud e integridad física, mental y social por problemas relacionados con la fertilidad.

En lo que sigue se abordará el problema propuesto desde la perspectiva del Derecho Internacional de los Derechos Humanos, con el fin de identificar los argumentos que permiten delimitar las obligaciones del Estado frente al acceso a técnicas de reproducción asistida a las personas que no las pueden garantizar con sus propios recursos.

\section{Los derechos sexuales $y$ reproductivos} en el Derecho Internacional de los Derechos

\section{Humanos}

El reconocimiento de los derechos sexuales y reproductivos en el contexto internacional ha tenido un desarrollo lento durante casi medio siglo. El primer referente de los derechos sexuales y reproductivos como derechos humanos se encuentra en la Conferencia Mundial de Derechos Humanos de Teherán (1968). En dicho foro se determinó que "los padres tienen el derecho humano fundamental de determinar libremente el número de sus hijos y los intervalos entre los nacimientos". Si bien el reconocimiento hecho en tal escenario establece un precedente sobre la protección que deben ejercer los Estados frente a las injerencias arbitrarias en la vida privada de las personas en su esfera reproductiva, es posible afirmar que fueron los diferentes mo- 
vimientos feministas surgidos desde la década del 60 los que, a través de la reivindicación de los principios de igualdad y no discriminación y bajo el postulado mediante el cual exigían que el "Estado no solo no podía interferir su libertad, sino que debía garantizar las condiciones para ejercerla", permitieron que se forjaran las bases para el posterior surgimiento de los derechos sexuales y reproductivos.

La Convención para la Eliminación de todas las formas de Discriminación contra la Mujer (1979) fue una batalla ganada por los movimientos feministas del siglo pasado en el ámbito jurídico, dicho tratado dio desarrollo a una de las principales premisas que formarán el corpus iuris para la protección de los derechos sexuales y reproductivos. Así, el artículo 16, literal e) de la Convención establece que los Estados tienen la obligación de asegurar tanto a hombres como mujeres "los mismos derechos a decidir libre y responsablemente el número de sus hijos y el intervalo entre los nacimientos y a tener acceso a la información, la educación y los medios que les permitan ejercer estos derechos". El anterior precepto desarrolla el derecho a fundar una familia, el cual había sido ya implementado en otros tratados y convenios de manera más general, como el Pacto Internacional de Derechos Civiles y Políticos, la Convención Americana de Derechos Humanos y el Convenio Europeo para la Protección de los Derechos Humanos y de las Libertades Fundamentales.

Es importante en este punto mencionar que si bien los derechos sexuales y los derechos reproductivos frecuentemente son mencionados en conjunto, estos tienen connotaciones diferentes. Así lo ha expresado la Corte Constitucional de Colombia, la cual entiende que "sexualidad y reproducción son dos ámbitos diferentes en la vida del ser humano, ya que la primera no debe ser entendida solamente como un medio para lograr la segunda" (Sentencia T-732, 2009). Así, dentro de los derechos sexuales podemos encontrar el derecho a decidir libremente sobre el propio cuerpo y sexualidad; derecho a decidir libremente con quién, cómo y cuándo ejercer la propia sexualidad; derecho a los servicios de salud sexual y derecho a la igualdad de oportunidades. Por otro lado, dentro de los derechos reproductivos se encuentra el ya mencionado derecho a decidir de forma libre y responsable el número y espaciamiento de los hijos e hijas; derecho a decidir libremente con quién, cuándo y cómo tener hijos e hijas; derecho a una educación oportuna e integral en salud reproductiva; derecho a acceder a servicios de aborto legal, seguro y de calidad y derecho a los servicios de salud reproductiva, libres de discriminación y de violencia. Es en esta segunda categoría donde se han desarrollado dos aspectos bien establecidos tanto en el Sistema Universal como Interamericano para la Protección de los Derechos Humanos, a saber, la autonomía y la salud reproductiva, que garantizan el acceso de las personas a técnicas y tratamientos de reproducción asistida.

En el año 1994 durante la Conferencia Mundial sobre Población y Desarrollo se incluyó por primera vez la salud sexual y reproductiva como un verdadero compromiso frente al bienestar de las personas y la garantía de sus derechos. En dicha oportunidad se determinó que: 
La salud reproductiva es un estado general de bienestar físico, mental y social, y no de mera ausencia de enfermedades o dolencias, en todos los aspectos relacionados con el sistema reproductivo y sus funciones y procesos. En consecuencia, la salud reproductiva entraña la capacidad de disfrutar de una vida sexual satisfactoria y sin riesgos y de procrear, y la libertad para decidir hacerlo o no hacerlo, cuándo y con qué frecuencia. Esta última condición lleva implícito el derecho del hombre y la mujer a obtener información y de planificación de la familia de su elección, así como a otros métodos para la regulación de la fecundidad que no estén legalmente prohibidos, y acceso a métodos seguros, eficaces, asequibles y aceptables, el derecho a recibir servicios adecuados de atención de la salud que permitan los embarazos y los partos sin riesgos y den a las parejas las máximas posibilidades de tener hijos sanos.

En el año 2000, el Comité de Derechos Económicos, Sociales y Culturales, en su Observación General No. 14 sobre el derecho al disfrute del más alto nivel posible de salud, determinó que:

La mujer y el hombre están en libertad para decidir si desean reproducirse y en qué momento, y tienen el derecho de estar informados y tener acceso a métodos de planificación familiar seguros, eficaces, asequibles y aceptables de su elección, así como el derecho de acceso a los pertinentes servicios de atención de la salud.

Estos estándares internacionales llevan a la conclusión que existe un vínculo irresistible entre estos dos aspectos, la salud y la autonomía reproductiva, por cuanto el no asegurar el libre ejercicio de esta última, necesariamente devendrá en una afectación a la primera. Esta consecuencia negativa en las libertades de las personas, a pesar de los esfuerzos por parte de la comunidad internacional para evitar su concreción, se evidencia con mayor ocurrencia, y especialmente en América Latina (Siverino-Bavio, 2012), en casos de personas con infertilidad. Es por esto que el precedente más significativo que ha consolidado el surgimiento de las técnicas de reproducción asistida como garantías para el ejercicio de los derechos reproductivos, es el reconocimiento por parte de la Organización Mundial de la Salud (2009) de la Infertilidad como "una enfermedad del sistema reproductivo definida como la incapacidad de lograr un embarazo clínico después de 12 meses o más de relaciones sexuales no protegidas". Dicha posición es respaldada por diversos estudios que muestran cómo la infertilidad genera:

... severas consecuencias sociales, económicas y de salud en los habitantes de los países en desarrollo; los efectos físicos, emocionales y psicosociales negativos en la salud pueden no ser fácilmente detectables pero ya se han documentado (...) La infertilidad es una condición individual y social, un individuo con infertilidad sufre de un cuerpo enfermo, una identidad humana sin cumplirse y presenta trastornos en sus relaciones con impacto social, político y económico. (McDonald, 2004)

Como respuesta a las necesidades de dicha población y con el fin de asegurar la garantía de los derechos sexuales y reproductivos de las personas, Estados como Argentina, y en menor medida Colombia recientemente, han venido 
reconociendo su obligación de brindar acceso a técnicas de reproducción asistida, mediante sus sistemas de salud a personas que de manera natural no pueden lograr dicho propósito. Estos avances en materia reproductiva se han materializado gracias al desarrollo que en términos de derechos humanos se ha venido gestando en el continente americano. Tal es el caso de la sentencia en el caso Artavia Murillo vs. Costa Rica de la Corte Interamericana de Derechos Humanos, con sede en dicho país, la cual ha sentado un precedente sobre las obligaciones concretas que en material de salud sexual y reproductiva tienen los Estados firmantes de la Convención Americana de Derechos Humanos.

En dicha ocasión, la Corte estudió el caso de una decisión del Tribunal Constitucional de Costa Rica que dejó sin efectos jurídicos el acto administrativo reglamentario sobre la práctica de técnicas de reproducción asistida y resolvió prohibir su implementación en el territorio costarricense. Como resultado, la Corte Interamericana desarrolló el derecho a la autonomía reproductiva, con base en los derechos a la vida privada y a la integridad personal expresamente protegidos por el instrumento referenciado. Específicamente, la Corte Interamericana de Derechos Humanos reconoció que:

... los derechos a la vida privada y a la integridad personal se hallan también directa e inmediatamente vinculados con la atención de la salud. La falta de salvaguardas legales para tomar en consideración la salud reproductiva puede resultar en un menoscabo grave del derecho a la autonomía y la libertad reproductiva. Existe por tanto una conexión entre la autonomía personal, la libertad reproductiva y la integridad física y psicológica (Caso Artavia Murillo vs. Costa Rica, 2012).

En esta oportunidad, que representa un valioso aporte para el desarrollo de los derechos sexuales y reproductivos, dicho tribunal determinó que prohibir la implementación de dichas técnicas constituía una vulneración del derecho a la vida privada y a la integridad personal, más aún cuando las personas tienen, en igualdad de condiciones, el derecho a acceder a las nuevas tecnologías que redunden en un beneficio para su salud, en este caso, su salud reproductiva. En palabras del Tribunal Regional:

Del derecho de acceso al más alto y efectivo progreso científico para el ejercicio de la autonomía reproductiva y la posibilidad de formar una familia se deriva el derecho a acceder a los mejores servicios de salud en técnicas de asistencia reproductiva, y, en consecuencia, la prohibición de restricciones desproporcionadas e innecesarias de iure o de facto para ejercer las decisiones reproductivas que correspondan en cada persona. (Resaltado fuera de texto) (Caso Artavia Murillo vs. Costa Rica, 2012).

Esta decisión servirá posteriormente a la Corte Constitucional colombiana para determinar si las restricciones al acceso a técnicas de reproducción asistida en el Sistema General de Seguridad Social en Salud para aquellas personas que por su infertilidad no puedan ejercer sus derechos reproductivos de manera natural, resulta desproporcional e innecesaria en el marco del Bloque de Constitucionalidad de Colombia.

De todo lo expuesto, es posible afirmar que 
los derechos sexuales y reproductivos, si bien han sido derechos de reciente desarrollo, en la actualidad se constituyen como verdaderas garantías para el ejercicio de las libertades más fundamentales de los seres humanos, como la igualdad y no discriminación, así como para el respeto de la dignidad humana, obligación inderogable para el Estado colombiano.

\section{La jurisprudencia de la Corte Constitucio- nal frente a los derechos sexuales y reproduc-} tivos y el acceso a TRA

Como es de suponerse, así como los derechos sexuales y reproductivos tuvieron un lento desarrollo en la esfera del Derecho Internacional de los Derechos Humanos, en el ámbito constitucional colombiano no corrieron con diferente suerte. Es posible afirmar que solo hasta la Constitución Política de 1991 se comenzó a dar forma a un cuerpo normativo sólido de protección de estos derechos.

La necesidad de implementar las teorías social-demócratas surgidas en Europa continental, lo cual condujo a la inevitable constitucionalización del Derecho, desencadenó todo un desarrollo progresivo de los principios de igualdad y no discriminación. Esto llevó a la reivindicación de ciertos derechos que gracias a la laicización del Estado han sido reconocidos por la Corte Constitucional colombiana, producto igualmente de este cambio estructural, como es el caso de los derechos de las minorías étnicas y en razón del género y por supuesto, los derechos sexuales y reproductivos. Así lo ha entendido dicho Tribunal, quien en el año 2012 insistió en que "tanto la Constitución de 1991 como varias normas pertenecientes al Bloque de Constitucionalidad reconocieron esta categoría de derechos y les dieron la naturaleza de fundamentales" (C. Const. Sentencia T-627/2012).

En dicha oportunidad, la alta Corte reiteró que "con fundamento en la Constitución, la jurisprudencia constitucional y los tratados internacionales sobre derechos humanos ratificados por Colombia, es posible afirmar que los derechos reproductivos reconocen y protegen (i) la autodeterminación reproductiva y (ii) el acceso a servicios de salud reproductiva". Dicho precedente obedece a la doctrina internacional anteriormente expuesta, la cual permite afirmar que el Estado tiene la obligación de emprender acciones positivas para con las personasque ven disminuido el ejercicio de sus derechos a la autonomía en conexidad con la salud reproductiva, como puede ser, brindar acceso a las técnicas de reproducción asistida mediante el Sistema General de Seguridad Social en Salud de Colombia.

Sin embargo, la posición de la Corte Constitucional no siempre ha sido la misma, por tal motivo, es importante hacer referencia al desarrollo jurisprudencial sobre el acceso a dichas técnicas como garantía para la protección de los derechos fundamentales de las personas con problemas de fertilidad, recorrido que culminará con la Sentencia T-274 de 2015, la cual puede ser identificada no solo como una sentencia hito en materia de garantía al acceso a las TRA sino

como un precedente para el desarrollo de los conceptos de autonomía y salud reproductiva en Colombia. 
Es imprescindible mencionar que la Corte Constitucional durante todo el desarrollo jurisprudencial sobre la materia, ha admitido que la limitación a los servicios prestados mediante el Plan Obligatorio de Salud es una restricción constitucionalmente admisible, atendiendo al principio de universalidad que rige el Sistema de Seguridad Social en Salud, postura evidenciada en prácticamente todas las sentencias que se referenciarán a continuación.

El primer referente en la jurisprudencia constitucional sobre el acceso a tratamientos contra la infertilidad en Colombia es la Sentencia T-1104 de 2000. En dicha oportunidad la Alta Corte estableció que los derechos de carácter prestacional como el derecho a la salud, solo podían ser tutelados cuando existiese "una relación de conexidad entre la garantía del derecho prestacional y la eficacia del derecho fundamental" (C. Const. Sentencia T-1104/2000), lo cual en la práctica se resumía a un análisis de conexidad entre el derecho a la salud y el derecho a la vida. Sin embargo, en esa misma jurisprudencia la Corte determinó que, desde un punto de vista bastante restringido, el derecho a la vida en condiciones dignas no se veía vulnerado por la inaccesibilidad a servicios médicos contra la infertilidad, por tal motivo, era improcedente la acción de tutela para exigir la práctica de dichos servicios. Asimismo, el Alto Tribunal acotó en dicha oportunidad que:

El orden axiológico de la Carta de 1991 mal podría sobreponer el goce de un derecho de segunda generación -como lo es el de hacer posible un embarazo de imposible viabilidad sin la interven- ción positiva estatal_, al derecho fundamental a la vida de una persona cuya patología la pone en riesgo de muerte, verbigracia un caso de urgente trasplante de hígado (C. Const. Sentencia T-1104/ 2000).

Desde la perspectiva de los derechos humanos, la Corte Constitucional yerra de manera arbitraria al intentar jerarquizar los derechos que pondera, atendiendo a una división clásica de los mismos, olvidando que, si bien el constitucionalismo contemporáneo determinó una distinción entre los derechos de acuerdo a su desarrollo histórico, la doctrina internacional sobre derechos humanos reconoce que son atributos propios de los derechos humanos: su interdependencia, es decir, la necesaria relación entre ellos para su efectiva realización y por otro lado, su indivisibilidad, es decir, la negación a cualquier intento de categorización o jerarquización de los mismos (Altemir, 2001). Esto implica que ... los Estados no están autorizados a proteger o garantizar una determinada categoría de derechos en contravención de otra, sino que todos los derechos humanos merecen la misma atención y urgencia (Serrano \& Vásquez, 2011). Sin embargo, esta postura será modificada por la Corte Constitucional, especialmente, desde el desarrollo del derecho a la salud, optando por una posición cada vez más garantista.

Durante los siguientes años el Alto Tribunal desde un enfoque preponderante del derecho a la salud y obviando la amplia argumentación desde una perspectiva de los derechos sexuales y reproductivos, estableció que, excepcionalmente, las personas con infertilidad podían acceder a 
tratamientos contra la infertilidad cuando (i) se pretenda garantizar la continuidad en la prestación del servicio de salud (C. Const. Sentencia T-946/2002), (ii) se requiere la práctica de exámenes diagnósticos para precisar una condición de salud de una mujer asociada a la infertilidad (C. Const. Sentencia T-229/2009) y (iii) cuando se requiera la práctica de tratamientos integrales en pacientes que padecen una enfermedad que afecta su aparato reproductor (C. Const; Sentencia T-752/2009). Estas subreglas, en apariencia, garantizaban de manera excepcional el derecho a la salud de las personas con infertilidad pero, en su materialización, su efectividad se hizo inocua para el acceso a técnicas de reproducción asistida pues es necesario reconocer plenamente no solo la afectación al derecho a la salud, como lo entendía la Corte, sino entender también la afectación de los derechos a la autonomía y salud reproductiva, los cuales se encuentran estrechamente relacionados con otras garantías como el derecho a la vida privada, al desarrollo de la personalidad y a la integridad personal.

Es importante mencionar que en el año 2014 la Corte Constitucional en su Sentencia T-528, si bien mantiene su posición frente a la improcedencia de la acción de tutela para otorgar acceso a técnicas de reproducción asistida mediante el Sistema General de Seguridad Social en Salud, hace un estudio de Derecho Comparado y del Derecho Internacional de los Derechos Humanos concluyendo que efectivamente existe un déficit en la protección de los derechos sexuales y reproductivos en Colombia. Por este motivo, exhorta: ...al Gobierno Nacional, por conducto del Ministerio de Salud y Protección Social y la Dirección de Regulación de Beneficios, Costos y Tarifas del Aseguramiento en Salud, para que realice la revisión de la situación que tienen que enfrentar las personas que padecen de infertilidad y no cuentan con recursos económicos para costear los tratamientos de reproducción humana asistida, entre ellos, la fertilización in vitro, e inicie una discusión pública y abierta de la política pública que incluya en la agenda la posibilidad de ampliar la cobertura del Plan Obligatorio de Salud a dichas técnicas científicas. (C. Const. Sentencia $\mathrm{T}-528 / 2014)$

Esta decisión es en esencia un avance a la discusión por cuanto advierte que la exclusión de las técnicas de reproducción asistida en el Plan Obligatorio de Salud obedece a la inobservancia de los derechos sexuales y reproductivos, no solo como verdaderos derechos humanos, sino como una consideración de gran relevancia en las políticas públicas sobre salud por parte del Estado colombiano. Además menciona que la progresividad del derecho a la salud como un derecho de carácter prestacional no puede ser un argumento suficiente para paralizar el desarrollo de dicho derecho y resalta que teniendo en cuenta que:

... el tiempo que ha transcurrido desde la adopción de la Constitución Política de 1991, el desarrollo paulatino del contenido prestacional del derecho a la salud, la progresividad de los sustentos teóricos y conceptuales de la jurisprudencia constitucional en el tema y los cambios legislativos, que ya incorporan la regulación del derecho 
fundamental a la salud en un proyecto de ley estatutaria; la Sala observa que la salud reproductiva es un aspecto de este derecho en donde puede reconocerse una insuficiencia de regulación y una opacidad de la jurisprudencia de la Corporación.

\section{(C. Const. Sentencia T-528/2014)}

No obstante, es la Sentencia T-274 de 2015 la que viene a modificar de manera trascendental la línea jurisprudencial de la Corte Constitucional sobre la protección de los derechos sexuales y reproductivos mediante el acceso a técnicas de reproducción asistida. En dicha oportunidad, la Corte reconoce que hasta aquel momento el análisis en los casos de acciones de tutela donde se solicitaba acceso a técnicas de reproducción asistida, se había centrado en la recuperación de la salud, es decir, hasta ese momento había hecho un análisis estricto del derecho a la salud. Sin embargo, de manera decisiva opta por enfocar la discusión, esta vez, desde:

una perspectiva diferente hasta ahora no examinada ni valorada en la jurisprudencia constitucional: la de los derechos a la igualdad y no discriminación, al libre desarrollo de la personalidad y a conformar una familia, su relación con la garantía de los derechos sexuales y reproductivos y su protección a través del sistema de seguridad social en salud. (C. Const. Sentencia T-274/2015)

Dentro del vasto análisis hecho por la Corte desde este nuevo enfoque, concluye esta que si bien la exclusión de las TRA es constitucionalmente admisible, la inaccesibilidad a dichas técnicas en cualquier caso puede generar la vulneración de garantías fundamentales. Afirma la Corte que si bien la inaccesibilidad puede no afectar directamente la vida e integridad de las personas, en todo caso no deja de ser una patología, según lo ha reconocido la propia Organización de la Salud, que sí atenta contra el derecho a la igualdad, entendido desde tres perspectivas diferentes" (C. Const. Sentencia T-274/2015). Esto, por cuanto la falta de acceso a técnicas que propendan la fertilidad y en últimas, la fecundación, no permite el desarrollo ni ejercicio de la autonomía reproductiva en condiciones de igualdad, en razón del género por cuanto dichas tecnologías son especialmente utilizadas sobre el cuerpo de la mujer; en razón de la discapacidad, en el entendido que la infertilidad puede ser vista como una y así ha sido asumida por la Corte Interamericana de Derechos Humanos y ahora la Corte Constitucional; y en razón de la condición socioeconómica toda vez que al no poseer la capacidad económica para asumir los altos costos de las técnicas, las personas de escasos recursos ven limitadas las posibilidades de fundar una familia a una única opción, la cual sería la adopción cuando "esta debe ser solamente una de varias opciones entre las cuales pueda escoger el paciente o la pareja, mas no su única alternativa” (C. Const. Sentencia T-274/2015).

Por otro lado, la Corte Constitucional acoge de manera integral el análisis que del derecho a la autonomía reproductiva hace la Corte Interamericana de Derechos Humanos en el caso Artavia Murillo vs. Costa Rica por encontrar una estrecha relación con los derechos al libre desarrollo de la personalidad y a constituir una familia. Al respecto, la Corte Constitucional afirma que: 
“existe un vínculo estrecho entre la autonomía personal, la libertad reproductiva y la integridad física y psicológica, este último conectado, a su vez, con el derecho a la salud, debido a que '[s] iendo la preservación de las condiciones físicas, psicológicas y espirituales de la persona objeto de protección del derecho fundamental a la integridad personal existe una estrecha relación entre este derecho y el derecho a la salud [pues este último] protege igualmente la preservación de la integridad de la persona humana, no solo frente a agresiones humanas sino también frente a todo tipo de agentes naturales o sociales. (C. Const. Sentencia T-636/2001)

Estos argumentos, llevan a la Corte a establecer unas nuevas subreglas aplicables para el acceso a técnicas de reproducción asistida en casos de infertilidad. A criterio de la Corte, es procedente la acción de tutela para acceder a dichas técnicas cuando (i) Se vulnere el derecho a la salud reproductiva, igualdad, libre desarrollo de la personalidad y a conformar una familia,

(ii) El tratamiento no tenga sustituto dentro del POS, (iii) El paciente no tenga la capacidad económica para sufragar los costos del tratamiento. Sin embargo, deberá hacer una contribución económica que en todo caso no podrá afectar su mínimo vital, (iv) el tratamiento sea autorizado por un médico de la EPS. Si dicha autorización procede de un particular se deberá conformar una junta médica por parte de la EPS y (v) se hayan estudiado las condiciones personales de viabilidad en cada caso.

Estas subreglas armonizan el problema jurídico en torno a la procedencia de la acción de tutela para acceder a tratamientos mediante técnicas de reproducción asistida con las subreglas que para el acceso a medicamentos, procedimientos y elementos no POS ya se habían propuesto con miras a proteger el derecho fundamental a la salud.

\section{CONCLUSIONES}

El artículo que se presenta, aborda en primer lugar el tema de la garantía del acceso a las técnicas de reproducción asistida para personas con problemas de fertilidad como una forma de garantizar los derechos sexuales y reproductivos. En esa medida se puede sostener que la imposibilidad de acceder a las TRA constituye una negación a estos derechos y a los derechos de libertad, en la medida en que la opción de la maternidad y paternidad están plenamente protegidos en las libertades fundamentales.

La Corte Constitucional de Colombia ha desarrollado una amplia jurisprudencia para proteger el derecho a la salud en sentido integral, como salud mental, física y social, reconociendo la procedencia de la acción de tutela para la protección de este derecho, cuando los peticionarios que no pueden costear tratamientos, medicamentos, procedimientos o exámenes de diagnóstico excluidos del POS vean vulnerados sus derechos ante la imposibilidad de acceder a ellos. Pese a esto, la Corte no había brindado la misma protección cuando se trataba de personas con problemas de fertilidad, actuando de manera incoherente frente al concepto de salud adoptado por la Corte y frente a la protección del derecho a la salud que les asiste a quienes no pueden, por 
medios naturales, disfrutar del derecho a elegir el número de hijos que quieren tener y con ello a la libertad y salud reproductiva.

El marco internacional de los derechos humanos permite afirmar que las razones de la Corte Constitucional para negar la protección mediante acción de tutela a quienes solicitaban el acceso a técnicas de reproducción asistida, antes de la Sentencia T-274 de 2015, eran insostenibles, pues condicionaban el acceso a este derecho a razones económicas y desconocían la noción de la salud como derecho integral, pese a que en la jurisprudencia de salud, en otros casos ya se había integrado este concepto.

La Sentencia T-274 de 2015 constituye un gran avance en el reconocimiento de los derechos sexuales y reproductivos, especialmente en su faceta de salud reproductiva, al determinar que su garantía afecta directamente otros derechos y que por lo tanto, debe garantizarse. La Corte reconoce que la reproducción no es un simple anhelo personal que en nada afecta la vida ni la integridad; en su lugar, afirma que en concordancia con el principio de interdependencia de los derechos, la garantía de la autonomía reproductiva necesariamente redunda en garantía de los demás derechos, como pueden ser la salud, la integridad y claro está, la vida en condiciones dignas.

La decisión del 2015, que da un giro en la argumentación precedente de la Corte en materia de acceso a las TRA mediante acción de tutela, es coherente entonces con el marco de proteccióndel Derecho Internacional de los Derechos Humanos frente a la garantía de los derechos sexuales y reproductivos, y permite unificar su jurisprudencia que protege el derecho a la salud de forma integral en Colombia.

Es preciso anotar, que pese a que se celebre este avance, el país sigue en mora de ampliar la protección de estos derechos. En el Congreso se

radicó en septiembre de 2013 un proyecto de Ley que buscaba reconocer la infertilidad como enfermedad, conforme a los estándares internacionales existentes en materia de salud y definir criterios para la cobertura de esta condición por parte del Sistema de Salud del Estado, sin embargo su trámite no se realizó y fue archivado por tránsito de legislatura el 19 de junio de 2015.

La garantía de los derechos sexuales y reproductivos implica un mayor activismo a nivel legislativo, dado que la protección mediante acción de tutela tiene como límite, como en otros casos (Pabón \& Aguirre, 2009, pp.278-279), en primer lugar que los afectados no tengan conocimiento sobre sus derechos y la forma de acceder a ellos y en segundo lugar, que los jueces de instancia no apliquen las reglas jurisprudenciales previstas.

\section{REFERENCIAS}

Alessio, M. (s.f.). La inseminación asistida con donación de esperma y sus implicancias con relación al derecho del niño del niño a la identidad y a la salud. Buenos Aires, Argentina: Universidad Nacional de la Plata. Recuperado el 2015, 04, 28, Disponible en http://www.calp.org.ar/uploads/ docs/ALESSIO_PROCREACION_HUMANA_ASISTIDA_PUBLICACIONES_CALP_pdf.pdf 
Altemir, A. B. (2001). Universalidad, indivisibilidad e interdependencia de los derechos humanos a los cincuenta años de la Declaración Universal. En La protección internacional de los derechos humanos a los cincuenta años de la declaración universal (pp.13-36). Tecnos.

Álvarez, M. (s.f.). Avances jurisprudenciales en las nuevas tipologías familiares en Colombia. Medellín, Colombia: Universidad de Antioquia. Recuperado el 2015, 04, 22. Disponible en http://www.udea. edu.co/portal/page/portal/bibliotecaSedesDependencias/unidadesAcademicas/ FacultadDerechoCienciasPoliticas/BilbiotecaDiseno/Archivos/01_Documentos/avancesJurisprudenciales.pdf

Arango, M. (2013). Derechos sexuales y reproductivos. En N. Lacrampette (Ed.), Derechos Humanos y Mujeres: teoría y práctica. Recuperado de www.cdh.uchile.cl/ media/publicaciones/pdf/101.pdf

Bernal, J. (2013). "Reproducción asistida y filiación, tres casos". Opinión Jurídica, 12, 135 a 150. Universidad de Medellín, Medellín. Recuperado 2014, 09, 21. Disponible en http://www.scielo.org.co/pdf/ojum/ v12n24/v12n24a09.pdf

Cabaleri, D. (2014). Las técnicas de reproducción humana asistida: el debate en la doctrina jurídica. Buenos Aires, Argentina: Biblioteca Digital de la Universidad Católica de Argentina. Recuperada el 2014, 10, 01. Disponible en http://bibliotecadigital.uca.edu.ar/repositorio/contri- buciones/tecnicas-reproduccion-humanacabaleri.pdf

Canessa, R. (2008). Problemas jurídicos que plantean las técnicas de reproducción humana asistida, en la legislación civil peruana (Tesis de Magíster en Derecho). Universidad Nacional Mayor de San Marcos, Lima, Perú. Recuperado 2014, 10, 01. Disponible en http://cybertesis.unmsm.edu.pe/bitstream/cybertesis/1487/1/ canessa_vr.pdf

Cárdenas, R. (2013). Alcances del derecho a la salud en Colombia: una revisión constitucional, legal y jurisprudencial. Revista de Derecho, (40), 198-225. Recuperado el 2016, 01, 20. Disponible en: http://rcientificas.uninorte.edu.co/index.php/derecho/article/view/4855

Casado, M. (1997). Reproducción humana asistida: los problemas que suscita desde la bioética y el derecho. Barcelona, España: SR. Recuperado el 2014, 10,

28. Disponible en file:///C:/Users/Ang/ Downloads/25478-25402-1-PB.pdf

Conferencia Mundial de Derechos Humanos de Teherán (1968). Proclamación de Teherán. Teherán.

Conferencia Mundial sobre Población y Desarrollo (1994). Informe. El Cairo.

Convención para la Eliminación de todas las formas de Discriminación contra la Mujer (1979).

Corte Interamericana de Derechos Humanos. Caso Artavia vs. Costa Rica, 14 de julio de 2011. 
Cubillos J. (2013). Status Jurídico del embrión Humano. Argentina: Universidad Nacional de Cuyo, Mendoza. Recuperado el 2015, 04, 28. Disponible en http://bdigital.uncu.edu.ar/objetos_digitales/5218/ cubillosjuanmanuel.pdf

Durand, T. \& Gutiérrez, M. (1998). Tras las huellas de un porvenir incierto: del aborto a los derechos sexuales y reproductivos.

Famá, M. (2012). El derecho a la identidad del hijo concebido mediante técnicas de reproducción humana asistida en el proyecto del Código Civil y Comercial de la Nación. Lecciones y ensayos, 90, 171-195. Recuperado el 2015, 04, 28. Disponible en http://www.derecho.uba.ar/publicaciones/lye/revistas/90/fama.pdf

González, M. (2012). Los Derechos del Niño en la reproducción asistida. México D.F.: Universidad Autónoma de México. Recuperado el 2015, 04, 27. Disponible en http://biblio.juridicas.unam.mx/libros/7/3155/8.pdf

Gutiérrez, F. (2013). La fecundación in vitro como un Derecho Humano Reconocido en el Ordenamiento Internacional de los Derechos Humanos (Tesis para Optar por el Grado de Licenciado en Derecho). Universidad de Costa Rica, Costa Rica. Recuperado el 2014, 11, 02. Disponible en http://iij.ucr.ac.cr/sites/default/files/ documentos/t13-la_fecundacion_in_vitro_como_un_derecho_humano_reconocido.pdf
Instituto Interamericano de Derechos Humanos (2008). Reproducción asistida género $y$ derechos humanos en América Latina. Fondo de Población de las Naciones Unidas. Recuperado el 2015, 4, 28. Disponible en http://bioetica.flacso.org.ar/ $\mathrm{img} / \mathrm{pdf} /$ Florencia-Luna-Reproduccionasistida.pdf

Lamas, M. (2001). Del cuerpo a la ciudadanía. El feminismo y la despenalización del aborto en México. En M. Valdés (Comp.), Controversias sobre el aborto. (pp.221239). México, D.F.: Fondo de Cultura Económica.

Luna, F. (2013). Infertilidad en Latinoamérica. En busca de un nuevo modelo. Revista de Bioética y Derecho, (28), 33-47. Disponible en: http://www.ub.edu/fildt/revista/ pdf/rbyd28_art-luna.pdf

McDonald, E. (2004). A global perspective on infertility: An under recognized public health issue. Chapel Hill, North Carolina, Estados Unidos: University Center for International Studies, The University of North Carolina.

Monroy, J. P. (2013). "Técnicas de reproducción asistida y su incidencia en Colombia". Verba Juris, 30. Recuperado el 2015, 04, 22. Disponible en http://www.unilibre. edu.co/verbaiuris/30/tecnicas-de-reproduccion-asistida-y-su-incidencia-en-colomnia.pdf

Muttarrasso, F. (2009). Antecedentes internacionales de legislación sobre fertilización asistida. Buenos Aires: Universidad J.F. 
Kennedy. Recuperado el 2015, 04, 28. Disponible en http://www.bioeticaclinica. com.ar

Organización de las Naciones Unidas (2000). Comité de Derechos Económicos, Sociales y Culturales. Ginebra. Recuperado de www.acnur.org/t3/fileadmin/documentos/ bol/2001/1451.pdf

Organización Mundial de la Salud (2009). Glosario de terminología en Técnicas de Reproducción Asistida (TRA). Versión revisada y preparada por el International Committee for Monitoring Assisted Reproductive Technology (ICMART) y la Organización Mundial de la Salud $(O M S)$. Red Latinoamericana de Reproducción Asistida.

Pabón \& Aguirre (2009). La protección jurisprudencial a la maternidad en Colombia. $R e$ vista Diálogo de Saberes, Julio-Diciembre, 263-282.

Quincosa, R. (2010). Análisis sobre la importancia de legislar la reproducción asistida en México (Tesis de Maestría). Universidad La Salle, Mexico D.F. Recuperado el 2015, 04, 27. Disponible en http://www. ordenjuridico.gob.mx/Publicaciones/

Tesis2011/2Analisis_sobre_la_importancia_de_legislar_la_reproduccion_asistida_en_Mexico.pdf

Samper, P. (s.f.). La fecundación asistida en Colombia: realidad y norma. Bogotá D.C., Colombia: Universidad de los Andes. Recuperado el 2015, 04, 27. Disponible en https://derechoprivado.uniandes.edu.co/ components/com_revista/archivos/derechoprivado/pri378.pdf

Sánchez, A. (s.f.). Legislación y jurisprudencia constitucional en materia de reproducción asistida y protección constitucional de la familia en España. Barcelona, España: Universidad de Barcelona. Recuperado el 2015, 04, 28. Disponible en http://www.udea.edu.co/portal/page/ portal/bibliotecaSedesDependencias/ unidadesAcademicas/FacultadDerechoCienciasPoliticas/BilbiotecaDiseno/Archivos/01_Documentos/LegislacionYJurisprudenciaConst.pdf

Sánchez, O. (2013). Persona, Derecho y Familia: Fundamentos del derecho de familia. Congreso Internacional "La familia hoy derechos y deberes", en el Centro de $\mathrm{Ne}$ gocios y Comercio de la ciudad de México. México D.F. Recuperado el 2015, 05, 27. Disponible en https://www.scjn.gob. $\mathrm{mx} /$ conocelacorte/ministra/persona\%20 derecho\%20y\%20familia.pdf

Serrano, S. \& Vásquez, L. (2011). Los principios de universalidad, interdependencia, indivisibilidad y progresividad, apuntes para su aplicación práctica. La reforma constitucional de derechos humanos: Un nuevo paradigma. México D.F.: Universidad Nacional Autónoma de México.

Siverino-Bavio, P. (2012). Una mirada desde la bioética jurídica a las cuestiones legales sobre la infertilidad en el Perú. Revista Peruana de Ginecología y Obstetricia. 
Suárez, A. (s.f.). Reproducción humana asistida y filiación en el derecho de familia colombiano. Revista virtual Camino del hallazgo y del juicio. Recuperado el 2014, 10, 01. Disponible en http://numanterioresviei.usta.edu.co/articulos/edi6/articulos/ reproducion-humana-asistidaok.pdf

Terribas, N. (2004). Procreación asistida y mediación en familia. Medellín, Colombia: Universidad de Antioquia. Recuperada el 2015, 04, 27. Disponible en http://www.udea.edu.co/portal/page/ portal/bibliotecaSedesDependencias/ unidadesAcademicas/FacultadDerechoCienciasPoliticas/BilbiotecaDiseno/Archivos/01_Documentos/avancesCientificosYJuridicosProcreacion.pdf

Zurriaráin, R. (2011). Técnicas de reproducción humana asistida: determinación legal de la filiación y usuarias en el Derecho Comparado. Universidad de La Rioja. Recuperado el 2015, 04, 22. Disponible en www.unirioja.es/dptos/dd/redur/numero8/barber.pd

\section{JURISPRUDENCIA}

Colombia. Corte Constitucional. Sentencia T1104 de 2000.

Colombia. Corte Constitucional. Sentencia T946 de 2002.

Colombia. Corte Constitucional. Sentencia T229 de 2009.

Colombia. Corte Constitucional. Sentencia T752 de 2009.

Colombia. Corte Constitucional. Sentencia T627 de 2012.

Colombia. Corte Constitucional. Sentencia T528 de 2014.

Colombia. Corte Constitucional. Sentencia T274 de 2015.

Colombia. Corte Constitucional. Setencia C-668 de 28 de junio 2005. M.P. Álvaro Tafur.

Colombia. Corte Constitucional. Setencia T-732 de 15 de octubre de 2009. M.P. Humberto Antonio Sierra Porto. 